\title{
Gene Expression Signatures of Peripheral Blood Mononuclear Cells during the Early Post-Transplant Period in Patients Developing Cardiac Allograft Vasculopathy
}

\author{
Khurram Shahzad, ${ }^{1}$ Martin Cadeiras, ${ }^{2}$ Sarfaraz Memon, ${ }^{1}$ Barry Zeeberg, ${ }^{3}$ Tod Klingler, ${ }^{4}$ \\ Anshu Sinha, ${ }^{1}$ Esteban G. Tabak, ${ }^{5}$ Sreevalsa Unniachan, ${ }^{1}$ and Mario C. Deng, 6 \\ ${ }^{1}$ Division of Cardiology, Department of Medicine, College of Physicians and Surgeons, Columbia University, New York, \\ NY 10032, USA \\ ${ }^{2}$ Division of Cardiology, Department of Medicine, University of Alabama at Birmingham, Birmingham, AL 35294, USA \\ ${ }^{3}$ Genomics and Bioinformatics Group, Laboratory of Molecular Pharmacology, National Cancer Institute, National Institutes of Health, \\ Bethesda, MD 20892, USA \\ ${ }^{4}$ XDx Inc., Brisbane, CA 94005, USA \\ ${ }^{5}$ Courant Institute of Mathematical Science, New York University, New York, NY 10012, USA \\ ${ }^{6}$ Cardiac Transplantation Research, Center for Advanced Cardiac Care, Columbia University College of Physicians \& Surgeons, \\ New York Presbyterian Hospital, PH Room 1291, 622 W 168th Street, New York, NY 10032, USA
}

Correspondence should be addressed to Mario C. Deng, md785@columbia.edu

Received 27 June 2010; Revised 18 September 2010; Accepted 27 September 2010

Academic Editor: Kazuhiko Yamada

Copyright (C) 2010 Khurram Shahzad et al. This is an open access article distributed under the Creative Commons Attribution License, which permits unrestricted use, distribution, and reproduction in any medium, provided the original work is properly cited.

\begin{abstract}
Background. Cardiac allograft vasculopathy (CAV) is a major cause of graft loss and death after heart transplantation. Currently, no diagnostic methods are available during the early post-transplant period to accurately identify patients at risk of CAV. We hypothesized that PBMC gene expression profiles (GEP) can identify patients at risk of CAV. Methods. We retrospectively analyzed a limited set of whole-genome PBMC microarrays from 10 post-transplant patients who did $(n=3)$ or did not $(n=7)$ develop advanced grade CAV during their long-term follow-up. We used significance analysis of microarrays to identify differentially expressed genes and High-Throughput GoMiner to assess gene ontology (GO) categories. We corroborated our findings by retrospective analysis of PBMC real-time PCR data from 33 patients. Results. Over 300 genes were differentially expressed (FDR < 5\%), and 18 GO-categories including "macrophage activation", "Interleukin-6 pathway", "NF-KappaB cascade", and "response to virus" were enriched by these genes (FDR $<5 \%$ ). Out of 8 transcripts available for RT-PCR analysis, we confirmed 6 transcripts (75.0\%) including FPRL1, S100A9, CXCL10, PRO1073, and MMP9 $(P<.05)$. Conclusion. Our pilot data suggest that GEP of PBMC may become a valuable tool in the evaluation of patients at risk of CAV. Larger prospectively designed studies are needed to corroborate our hypothesis.
\end{abstract}

\section{Introduction}

Cardiac allograft vasculopathy (CAV) is a major cause of graft loss and death after heart transplantation (HTx). Identification of surrogate makers for late cardiac allograft survival has been of major interest to improve long-term outcomes of HTx [1]. After HTx, alloantigens (including molecules from donor endothelium) are presented by antigen-presenting cells to the recipient's T-cells, often generating a differentiated inflammatory response. That response includes T-cells, B-cells, and a coordinated pattern of cytokine release. Cells of innate immunity (monocytederived macrophages) are also involved [1]. Non-antigenspecific perioperative events including microvascular insults may play pivotal roles related to subsequent development of CAV, probably related to ischemia reperfusion injury, advanced donor age, hyperlipidemia, depletion of arteriolar tissue plasminogen activator factor and systemic 
inflammation [1-8]. The inflammatory response culminates in migration of mononuclear cells through the coronary vascular endothelium and phenotypic switching of medial smooth muscle cells mediated by generation of growthpromoting cytokines. Those processes contribute to chronic damage of the coronary arteries of the transplanted heart. The result is the development of a diffuse, obliterative form of vasculopathy characterized by production of a "neointima" rich in vascular smooth muscle cells and extracellular matrix $[9,10]$. After the first year post-transplantation, $30 \%$ to $50 \%$ of patients have some evidence of CAV and after 5 years CAV is one of the leading causes of death with $<50 \% 1$-year survival rate in those with extended disease $[11,12]$.

Limited interventions have been shown to prevent, delay, or reverse CAV. While no definite well-validated surrogate marker for late cardiac allograft outcome is available, early detection of CAV represents the key strategy as an effective surrogate [1]. Early identification of CAV became possible with the introduction of intravascular ultrasound (IVUS) [13], but the technique is invasive, it is usually not initiated until at least one year post-transplantation, is expensive, and requires the use of nephrotoxic contrast agents. Noninvasive tests, including stress perfusion, dobutamine echocardiography, ultrafast tomography, and MRI have not proven to be sufficiently sensitive or specific to detect early stages of the disease $[14,15]$. Therefore, there are clear needs to explore and develop new options for the early evaluation of patients at risk of CAV.

Recently, gene expression profiles of peripheral blood mononuclear cells (PBMC) were used to identify patients with $[16,17]$ or without moderate and severe acute cellular cardiac allograft rejection [18] and patients at risk of antibody-mediated rejection [19].

Since the peripheral recirculation of recipient leukocytes after allo-endothelial-cell contact in the allograft may carry information about immune activation conducive to chronic rejection development, we hypothesized that gene expression profiles of PBMC obtained early after HTx carry molecular signatures that correlate with the future development of CAV.

\section{Materials and Methods}

2.1. Patients, Samples, and Microarrays. We analyzed a limited set of 41,000 gene expression profiles (wholegenome Microarray, Agilent Technologies, Wilmington, DE) obtained from patients included in a large multicenter study (Cardiac Allograft Rejection Gene Expression Observational [CARGO] study) that used microarrays to identify PBMC gene signatures of acute cellular cardiac allograft rejection [18]. Columbia University Medical Center (CUMC) contributed 121 patients. Out of the Columbia University cohort, independent whole genome PBMC samples from 10 patients were available. The study protocol was approved by the Institutional Review Board (IRB) of CUMC. PBMC were isolated from eight $\mathrm{mL}$ of venous blood using density gradient centrifugation (CPT, Becton-Dickinson, Franklin Lakes, NJ). Samples were frozen in lysis buffer (RLT, Qiagen, Valencia, CA) within $2 \mathrm{~h}$ of phlebotomy. Total RNA was isolated from each sample (RNeasy, Qiagen, Valencia, CA).
Whole genome gene expression profiling was performed on two-color Whole Human Genome 60-mer Oligo Microarrays (Agilent Technologies, Santa Clara, CA), which contain $41,000+$ unique transcripts. After the hybridization, data were extracted and Lowess normalized expression files were generously provided by the CARGO study sponsor (XDx Inc., Brisbane, CA). Filtering was done against background and only those probes with more than 1.5 -fold change were retained. Probes mapping to the same gene transcript were not averaged.

2.2. Cardiac Allograft Vasculopathy. Patients were eligible for the study if they were evaluated with angiography during their post-transplant course. CAV was defined as any evidence of disease in the angiography as evaluated by one of three expert interventional cardiologists who specialized in heart transplantation care at the Center for Interventional Vascular Therapy at Columbia University. Angiograms were classified as normal (no evidence of vasculopathy), mild (any grade of angiographic luminal stenosis less than 50\%), moderate (any angiographic luminal stenosis greater than $50 \%$ in a main vessel or two secondary branches), and severe (more than $50 \%$ in the left main or two main vessels). Patients with moderate or severe CAV were considered "advanced".

2.3. Statistical Analysis. Quantitative and qualitative clinical variables were compared by the Mann Whitney $U$-test, Chi-Square test, or Fischer-exact when appropriate using SPSS 11.5.1 (SPSS Inc., 2002). A $P$-value $<.05$ was regarded as significant. Gene expression of samples obtained from patients with advanced CAV was compared against those with normal or mild disease using independent, unpaired $t$-test, and Benjamini-Hochberg correction for multiple comparisons to estimate a false discovery rate (FDR) as implemented in significance analysis of microarrays (SAM) [20] after 1.5-fold change filtering. Genes were retained for further analyses if FDR $<5 \%$. Molecular function and biological processes associated with individual significant genes were determined using PANTHER (Protein ANalysis THrough Evolutionary Relationships) Classification System (http://www.pantherdb.org), a unique resource that classifies genes by their functions, using published scientific experimental evidence and evolutionary relationships to predict function even in the absence of direct experimental evidence. Quantitative, real-time PCR cycle thresholds (CT) for each group were compared with the Mann Whitney $U$-test. $P$ value $<.05$ was considered significant.

2.4. Hierarchical Clustering. Gene expression data were correlated and visualized in clustered heat maps [21] using the Genesis web interface ((http://carmaweb.genome.tugraz.at/ genesis/index2.html), Institute for Genomics and Bioinformatics, Graz University of Technology, Graz, Austria). Top 100 genes and microarray samples as well as differentially enriched GO categories and genes were clustered on the basis of co-occurrence of the differentially expressed genes using the Pearson metric and average linkage. 
TAble 1: Baseline clinical characteristics. Baseline Clinical Characteristics Comparing Microarray and PCR Study Populations.

(a)

\begin{tabular}{|c|c|c|c|c|c|c|}
\hline \multirow[b]{2}{*}{ Variable } & \multicolumn{3}{|c|}{ Microarray study $(N=10)$} & \multicolumn{3}{|c|}{ PCR study $(N=33)$} \\
\hline & $\begin{array}{l}\text { Normal or } \\
\text { mild }(N=7)\end{array}$ & $\begin{array}{l}\text { Advanced CAV } \\
\quad(N=3)\end{array}$ & $P$-value & $\begin{array}{c}\text { Normal or } \\
\text { mild }(N=28)\end{array}$ & $\begin{array}{c}\text { Advanced CAV } \\
\quad(N=5)\end{array}$ & $P$-value \\
\hline Time after HTx (days) & $232(81-298)$ & $42(41-96)$ & .067 & $81(7-171)$ & $55(41-167)$ & .643 \\
\hline Follow-up time (years) & $5.3(4.8-5.8)$ & $5.6(5.4-5.7)$ & .138 & $5.5(4.6-6.2)$ & $5.7(5.1-5.8)$ & .315 \\
\hline Male gender recipient & $6(85.7 \%)$ & $3(100 \%)$ & 1 & $21(75.0 \%)$ & $4(80 \%)$ & 1 \\
\hline Male gender donor & $6(85.7 \%)$ & $2(66.7 \%)$ & 1 & $20(71.4 \%)$ & $3(60 \%)$ & .627 \\
\hline Caucasian recipient & $5(71.4 \%)$ & $3(100 \%)$ & .585 & $21(63.6 \%)$ & $5(100 \%)$ & .903 \\
\hline Caucasian donor & $7(100 \%)$ & $1(33 \%)$ & .67 & $17(60.7 \%)$ & $2(40 \%)$ & .685 \\
\hline Recipient age & $55.6 \pm 8.2$ & $48.5 \pm 13.5$ & .383 & $52.6 \pm 105$ & $61.4 \pm 8$ & .173 \\
\hline Donor age & $31.7 \pm 11.7$ & $44.1 \pm 7.9$ & .183 & $32.8 \pm 13.0$ & $41.5 \pm 13.7$ & .226 \\
\hline ICM recipient & $5(71.4 \%)$ & $1(33.3 \%)$ & .11 & $14(50 \%)$ & $4(80 \%)$ & .267 \\
\hline LVAD & $3(42.9 \%)$ & $2(66.7 \%)$ & 1 & $7(21.2 \%)$ & $2(40 \%)$ & .597 \\
\hline Recipient CMV (+) & $2(28.6 \%)$ & $0(0.00 \%)$ & .53 & $11(33.3 \%)$ & $2(40 \%)$ & .347 \\
\hline Donor CMV $(+)$ & $3(42.9 \%)$ & $2(40.0 \%)$ & .7 & $14(50 \%)$ & $4(80 \%)$ & .445 \\
\hline Ischemic time (Min) & $188.7 \pm 65.1$ & $138 \pm 36.6$ & .229 & $183.9 \pm 49.2$ & $156 \pm 7$ & .208 \\
\hline Daclizumab induction & $7(100 \%)$ & $3(100 \%)$ & - & $24(85.7 \%)$ & $4(80 \%)$ & \\
\hline Prednisone & $7(100 \%)$ & $3(100 \%)$ & - & $28(100 \%)$ & $5(100 \%)$ & - \\
\hline Prednisone dose & $8.71 \pm 7.8$ & $13.3 \pm 2.9$ & .296 & $16.3 \pm 10.2$ & $13.0 \pm 4.5$ & .359 \\
\hline Maintenance Regimen & & & 1 & & & .295 \\
\hline Cyclosporine & $6(85.7 \%)$ & $3(100 \%)$ & & $24(85.7 \%)$ & $4(80 \%)$ & \\
\hline Tacrolimus & $1(14.3 \%)$ & $0(0.0 \%)$ & & $3(10.7 \%)$ & $0(0 \%)$ & \\
\hline Sirolimus & $0(0.0 \%)$ & $0(0.0 \%)$ & & $1(3.6 \%)$ & $1(20 \%)$ & \\
\hline Cyclosporine levels ( $\mathrm{ng} / \mathrm{ml})$ & $437.0 \pm 489$ & $225.3 \pm 26$ & .655 & $312.5 \pm 226$ & $229.25 \pm 22$ & .212 \\
\hline Tacrolimus levels (ng/ml) & $8.5 \pm 0.2$ & - & - & $11.7 \pm 2.5$ & - & - \\
\hline Sirolimus levels (ng/ml) & - & - & - & 6 & 5.6 & - \\
\hline Mycophenolate & $6(85.7 \%)$ & $3(100 \%)$ & 1 & $24(85.7 \%)$ & $5(100 \%)$ & 1 \\
\hline Azathioprine & $0(0.0 \%)$ & $0(0.0 \%)$ & - & $0(0.0 \%)$ & $0(0.0 \%)$ & - \\
\hline Simvastatin & $1(14 \%)$ & $1(33 \%)$ & 1.00 & $2(7 \%)$ & $0(0 \%)$ & 1.00 \\
\hline Cellular rejection (ISHLT) & & & .161 & & & .78 \\
\hline $0 \mathrm{R}$ & $4(57.1 \%)$ & $2(66.7 \%)$ & & $24(85.7 \%)$ & $4(80 \%)$ & \\
\hline $1 \mathrm{R}$ & $3(42.9 \%)$ & $0(0.0 \%)$ & & $4(14.3 \%)$ & $1(20 \%)$ & \\
\hline $2 \mathrm{R}$ & $0(0.0 \%)$ & $1(33.3 \%)$ & & $0(0 \%)$ & $0(0 \%)$ & \\
\hline \multicolumn{7}{|l|}{ New episodes of rejection } \\
\hline $1 \mathrm{R} / 1 \mathrm{~B}$ & $0(0-1)$ & $0(0-0)$ & .513 & $0(0-4)$ & $0(0-3)$ & .688 \\
\hline $2 \mathrm{R}$ & $1(0-1)$ & $1(0-1)$ & .789 & $0(0-2)$ & $0(0-1)$ & .375 \\
\hline $2 \mathrm{R} / 3 \mathrm{~A}$ & $0(0-1)$ & $0(0-0)$ & .513 & $0(0-2)$ & $0(0-3)$ & .898 \\
\hline
\end{tabular}

HTx: heart transplant; LVAD: left ventricular assist device; RT-PCR: real-time polymerase chain reaction.

(b)

\begin{tabular}{|c|c|c|c|c|c|c|c|c|c|}
\hline \multirow[b]{2}{*}{ Variable } & \multicolumn{3}{|c|}{ Normal or mild } & \multicolumn{3}{|c|}{ Advanced CAV } & \multicolumn{3}{|c|}{ Microarray versus PCR $(N=43)$} \\
\hline & $\begin{array}{l}\text { Microarray } \\
\quad(N=7)\end{array}$ & $\begin{array}{c}\text { RT-PCR } \\
(N=28)\end{array}$ & $P$-value & $\begin{array}{l}\text { Microarray } \\
(N=3)\end{array}$ & $\begin{array}{l}\text { RT-PCR } \\
(N=5)\end{array}$ & $P$-value & $\begin{array}{l}\text { Microarray } \\
(N=10)\end{array}$ & $\begin{array}{l}\text { RT-PCR } \\
(N=33)\end{array}$ & $P$-value \\
\hline $\begin{array}{l}\text { Time after HTx } \\
\text { (days) }\end{array}$ & $232(81-298)$ & $81(7-171)$ & .002 & $42(41-96)$ & $55(41-167)$ & .571 & $154.5 \pm 99.5$ & $76.4 \pm 47.5$ & .037 \\
\hline $\begin{array}{l}\text { Follow-up time } \\
\text { (years) }\end{array}$ & $5.3(4.8-5.8)$ & $5.5(4.6-6.2)$ & .433 & $5.6(5.4-5.7)$ & $5.7(5.1-5.8)$ & .451 & $5.3 \pm 0.3$ & $5.4 \pm 0.4$ & .583 \\
\hline
\end{tabular}


(b) Continued.

\begin{tabular}{|c|c|c|c|c|c|c|c|c|c|}
\hline \multirow[b]{2}{*}{ Variable } & \multicolumn{3}{|c|}{ Normal or mild } & \multicolumn{3}{|c|}{ Advanced CAV } & \multicolumn{3}{|c|}{ Microarray versus PCR $(N=43)$} \\
\hline & $\begin{array}{c}\text { Microarray } \\
(N=7)\end{array}$ & $\begin{array}{l}\text { RT-PCR } \\
(N=28) \\
\end{array}$ & $P$-value & $\begin{array}{c}\text { Microarray } \\
(N=3)\end{array}$ & $\begin{array}{l}\text { RT-PCR } \\
(N=5)\end{array}$ & $P$-value & $\begin{array}{l}\text { Microarray } \\
(N=10)\end{array}$ & $\begin{array}{l}\text { RT-PCR } \\
(N=33)\end{array}$ & $P$-value \\
\hline $\begin{array}{l}\text { Male gender } \\
\text { recipient }\end{array}$ & $6(85.7 \%)$ & $21(75 \%)$ & 1 & $3(100 \%)$ & $4(80 \%)$ & 1 & $9(90 \%)$ & $25(75.8 \%)$ & 659 \\
\hline $\begin{array}{l}\text { Male gender } \\
\text { donor }\end{array}$ & $6(85.7 \%)$ & $20(21.4 \%)$ & .648 & $2(66.7 \%)$ & $3(60 \%)$ & 1 & $8(80 \%)$ & $23(69.7 \%)$ & .698 \\
\hline $\begin{array}{l}\text { Caucasian } \\
\text { recipient }\end{array}$ & $5(71.4 \%)$ & $21(75 \%)$ & .781 & $3(100 \%)$ & $5(100 \%)$ & & $8(80 \%)$ & $26(78.8 \%)$ & .823 \\
\hline Caucasian donor & $7(100 \%)$ & $17(60.7)$ & .26 & $1(33.3 \%)$ & $2(40 \%)$ & .641 & $8(80 \%)$ & $19(57.6 \%)$ & .433 \\
\hline Recipient age & $55.6 \pm 8.2$ & $52.6 \pm 10.5$ & .43 & $48.6 \pm 13.5$ & $61.4 \pm 8.0$ & .393 & $53.5 \pm 9.8$ & $53.9 \pm 10.5$ & .916 \\
\hline Donor age & $31.7 \pm 11.6$ & $32.8 \pm 13.0$ & .888 & $44.1 \pm 7.9$ & $41.55 \pm 13.6$ & 1 & $35.4 \pm 11.8$ & $34.1 \pm 13.3$ & .559 \\
\hline ICM recipient & $5(71.4 \%)$ & $14(50 \%)$ & .941 & $1(33.3 \%)$ & $4(80 \%)$ & .293 & $6(60 \%)$ & $18(54.5 \%)$ & .668 \\
\hline LVAD & $3(42.9 \%)$ & $7(25 \%)$ & .31 & $2(66.7 \%)$ & $2(40 \%)$ & 1 & $5(50 \%)$ & $9(27.3 \%)$ & .252 \\
\hline $\begin{array}{l}\text { Recipient CMV } \\
(+)\end{array}$ & $2(28.6 \%)$ & $11(39.3 \%)$ & .523 & $0(0.0 \%)$ & $2(40 \%)$ & .449 & $2(20 \%)$ & $13(39.4 \%)$ & .291 \\
\hline Donor CMV $(+)$ & $3(42.9 \%)$ & $14(50 \%)$ & .822 & $2(66.7 \%)$ & $4(80 \%)$ & 1 & $5(50 \%)$ & $18(54.5 \%)$ & .904 \\
\hline $\begin{array}{l}\text { Ischemic times } \\
\text { (Min) }\end{array}$ & $\begin{array}{l}188.75 \pm \\
65.13\end{array}$ & $183.9 \pm 49.2$ & .706 & $138.0 \pm 36.6$ & $156.0 \pm 7$ & .786 & $167 \pm 57.5$ & $178.7 \pm 45.6$ & .495 \\
\hline $\begin{array}{l}\text { Daclizumab } \\
\text { induction }\end{array}$ & $7(100 \%)$ & $24(85.7 \%)$ & .562 & $3(100 \%)$ & $4(80 \%)$ & & $10(100 \%)$ & $28(84.8 \%)$ & .32 \\
\hline Prednisone & $7(100 \%)$ & $28(100 \%)$ & - & $3(100 \%)$ & $5(100 \%)$ & & $10(100 \%)$ & $33(100 \%)$ & \\
\hline Prednisone dose & $8.7 \pm 7.8$ & $16.3 \pm 10.2$ & .015 & $13.3 \pm 2.9$ & $13.0 \pm 4.5$ & .845 & $10.1 \pm 6.8$ & $15.79 \pm 9.5$ & .041 \\
\hline $\begin{array}{l}\text { Maintenance } \\
\text { regimen }\end{array}$ & & & .85 & & & .4 & & & .727 \\
\hline Cyclosporine & $6(85.7 \%)$ & $24(85.7 \%)$ & & $3(100 \%)$ & $4(80 \%)$ & & $9(90 \%)$ & $28(84.8 \%)$ & \\
\hline Tacrolimus & $1(14.3 \%)$ & $3(10.7 \%)$ & & $0(0.0 \%)$ & $0(0.0 \%)$ & & $1(10 \%)$ & $3(9.1 \%)$ & \\
\hline Sirolimus & $0(0.0 \%)$ & $0(0.0 \%)$ & & $0(0.0 \%)$ & $1(20 \%)$ & & $0(0.0 \%)$ & $2(6.1 \%)$ & \\
\hline $\begin{array}{l}\text { Cyclosporine } \\
\text { levels ( } \mathrm{ng} / \mathrm{ml})\end{array}$ & $437.0 \pm 489.4$ & $312.5 \pm 226$ & .686 & $225.3 \pm 26.6$ & $229.25 \pm 22.6$ & 1 & $357.6 \pm 386$ & $300.6 \pm 211$ & .47 \\
\hline $\begin{array}{l}\text { Tacrolimus levels } \\
\text { (ng/ml) }\end{array}$ & $8.5 \pm 0.2$ & $11.7 \pm 2.5$ & .083 & - & - & & $8.5 \pm 0.2$ & $11.7 \pm 2.5$ & .083 \\
\hline $\begin{array}{l}\text { Sirolimus levels } \\
(\mathrm{ng} / \mathrm{ml})\end{array}$ & - & 6 & & - & 5.6 & & - & $11.7 \pm 2.5$ & - \\
\hline Mycophenolate & $6(85.7 \%)$ & $24(85.7 \%)$ & 1 & $3(100 \%)$ & $5(100 \%)$ & & $9(90 \%)$ & $28(84.8 \%)$ & 1 \\
\hline Azathioprine & $0(0.0 \%)$ & $0(0.0 \%)$ & - & $0(0.0 \%)$ & $0(0.0 \%)$ & - & $0(0.0 \%)$ & $0(0.0 \%)$ & - \\
\hline Simvastatin & $1(14 \%)$ & $2(7 \%)$ & .499 & $1(33 \%)$ & $0(0 \%)$ & .375 & $2(20 \%)$ & $2(6 \%)$ & .226 \\
\hline $\begin{array}{l}\text { Cellular rejection } \\
\text { (ISHLT) }\end{array}$ & & & .123 & & & .315 & & & .127 \\
\hline $0 \mathrm{R}$ & $4(57.1 \%)$ & $24(85.7 \%)$ & & $2(66.7 \%)$ & $4(80 \%)$ & & $6(60 \%)$ & $28(84.8 \%)$ & \\
\hline $1 \mathrm{R}$ & $3(42.9 \%)$ & $4(14.3 \%)$ & & $0(0.0 \%)$ & $1(20 \%)$ & & $3(30 \%)$ & $5(15.1 \%)$ & \\
\hline $2 \mathrm{R}$ & $0(0.0 \%)$ & $0(0.0 \%)$ & & $1(33.3 \%)$ & $0(0.0 \%)$ & & $1(10 \%)$ & $0(0.0 \%)$ & \\
\hline \multicolumn{10}{|l|}{$\begin{array}{l}\text { New Episodes of } \\
\text { Rejection }\end{array}$} \\
\hline $1 \mathrm{R} / 1 \mathrm{~B}$ & $0(0-1)$ & $0(0-4)$ & .3 & $0(0-0)$ & $0(0-3)$ & .439 & $0(0-1)$ & $0(0-4)$ & .17 \\
\hline $2 \mathrm{R}$ & $1(0-1)$ & $0(0-2)$ & .59 & $1(0-1)$ & $0(0-1)$ & .217 & $1(0-1)$ & $0(0-2)$ & .32 \\
\hline $2 \mathrm{R} / 3 \mathrm{~A}$ & $0(0-1)$ & $0(0-2)$ & .4 & $0(0-0)$ & $0(0-3)$ & .439 & $0(0-1)$ & $0(0-3)$ & .235 \\
\hline
\end{tabular}

HTx: heart transplant; LVAD: left ventricular assist device; RT-PCR: real time polymerase chain reaction. 
2.5. Gene Ontology Analysis. We used High-Throughput GoMiner (HTGM) [22] (http://discover.nci.nih.gov/) to analyze the list of interesting genes in the context of gene ontology (GO) categories [23]. HTGM analyzes data from all microarrays in a study, provides diagnostics for data interpretation and visualization tools in terms of specialized clustered "heat maps" (also called clustered image maps, CIMs) [21]. Normally, the input to HTGM consists of a totalgenes file (representing the entire Microarray or a randomly generated whole genome seed) and a changed-genes file (representing the genes with altered expression) relevant to the study purpose. The output generated by HTGM includes a summary of the results, a matrix whose rows are categories and whose columns are names of changed gene for hierarchical clustering of experiments and categories, and a statistical summary for each category including one-sided Fisher exact $P$-value and an FDR. Hierarchical clustering of enriched categories and changed genes allows determining which categories achieved statistical significance by virtue of containing essentially the same set of changed genes.

\section{Results}

3.1. Patient Demographics. The baseline characteristics of the study population, including mean age, gender, race, diagnosis, mean cold ischemia time, induction strategy, antirejection prophylaxis, median follow-up time at the time that the microarrays were obtained, median followup time till the diagnosis of CAV, simvastatin use, and median number of rejection episodes (i.e., $1 \mathrm{R} / 1 \mathrm{~B}, 2 \mathrm{R}$, and $2 \mathrm{R} / 3 \mathrm{~A}$ ) requiring augmented immunosuppression are depicted in Tables 1(a) and 1(b) for the overall population and by study subgroups and sample method. All baseline clinical characteristics were comparable across groups in both microarray and PCR studies. Although the difference in timing of blood sample post-HTx between advanced CAV and normal/mild CAV groups in the microarray study population approached the defined level of significance $(P=$ $.06)$ but this difference was not significant in the PCR study population.

3.2. Microarray Analysis. Out of 10 patients who were studied with microarrays, 3 (30\%) had advanced CAV while $7(70 \%)$ had angiograms consistent with either absence of disease $(n=3,30 \%)$ or mild disease $(n=4,40 \%)$. Comparison of study samples for each study group is summarized in Table 1(a). One sample that had 2R (moderate) cellular cardiac allograft rejection was retained given the limited small Microarray dataset.

3.3. RT-PCR Analysis. There were 64 Quantitative, 253 transcript, Real-Time PCR samples available from our Center, obtained from 43 patients during the CARGO study [18]. Nine samples from 9 patients associated with ISHLT grade $3 \mathrm{~A} / 2 \mathrm{R}$ rejection and 1 sample without rejection grading information were excluded. Out of the remaining samples, 3 samples had no angiographic information, 1 sample had aneurismatic disease diagnosed at year 1 without further information in subsequent years, and 18 samples from
13 patients were repeated. For repeated samples, we choose the one closest to the median time from the transplant surgery to the date that all samples were obtained. Therefore, we retained 33 samples obtained from 33 patients for this analysis. Twenty-eight PCR samples (85\%) belonged to patients from the normal $(n=20)$ or mild disease $(n=$ 8 ) group, and 5 samples (15\%) belonged to patients with advanced CAV.

3.4. Gene Expression Profiles. Between-group comparisons identified 316 transcripts from 291 unique genes differentially expressed with FDR $\leq 5 \%$ : 182 transcripts (170 genes) were up-regulated and 134 transcripts (123 genes) were down-regulated. Top up- and down-regulated genes and their functions are depicted in (Table 2) and a clustered heat map of top 100 up- and down-regulated genes is shown in (Figure 1). A complete list of genes with their respective fold changes and false discovery rates is provided as supplementary material available online at doi:10.1155/2010/719696.

3.5. Gene Ontology Analysis. HTGM detected 18 changed GO categories (FDR $<5 \%$ ) enriched with differentially expressed genes (Table 3). Processes related to enriched GO categories included among others: macrophage activation, response to wounding, response to virus, response to biotic stimulus, interleukin-6 biosynthetic process, I-kappaB kinase NF-kappaB cascade, innate immune response, inflammatory response, regulation of interleukin- 6 biosynthetic process, defense response, positive regulation of interleukin- 6 biosynthetic process, activation of NF-kappaB-inducing kinase, and interleukin-6 production. Evaluation of the clustered image map indicates potential cross-talk among GO categories enriched by advanced CAV genes. Instances of cross-talk are of particular importance because they tie together categories that, based on prior knowledge, might be thought of as unrelated. A clustered heat map of differentially expressed genes and enriched gene ontology categories and cross-talk is shown in (Figure 2).

3.6. RT-PCR Validation. We compared the list of 291 candidate genes that we identified by SAM based on 5\% FDR for this exploratory study with the 252 candidate gene list of the available RT-PCR generated in the Discovery Phase of the CARGO study [18]. We found 8 transcripts (7 different genes) overlapping. The validation rate based on Mann Whitney $U$-test comparison between absence of, or mild CAV $(n=28)$ and advanced CAV $(n=5)$ was $75 \%$ (2 not validated out of 8 ). Validated genes included FPRL1 $(P=.005)$, S100A9 $(P=.005)$, CXCL10 transcript $1(P=.009)$, and CXCL10 transcript $2(P=.019)$, PRO1073 $(P=.045)$, MMP9 $(P=.045)$. Nonvalidated genes included ENO1 $(0.083)$ and FAS $(P=.104)$. SAM-based differentially expressed genes and the corresponding RT-PCR gene $P$ values showed good concordance (Table 4).

\section{Discussion}

A gene signature is the group of genes in a type of cell whose combined expression pattern is a unique characteristic 

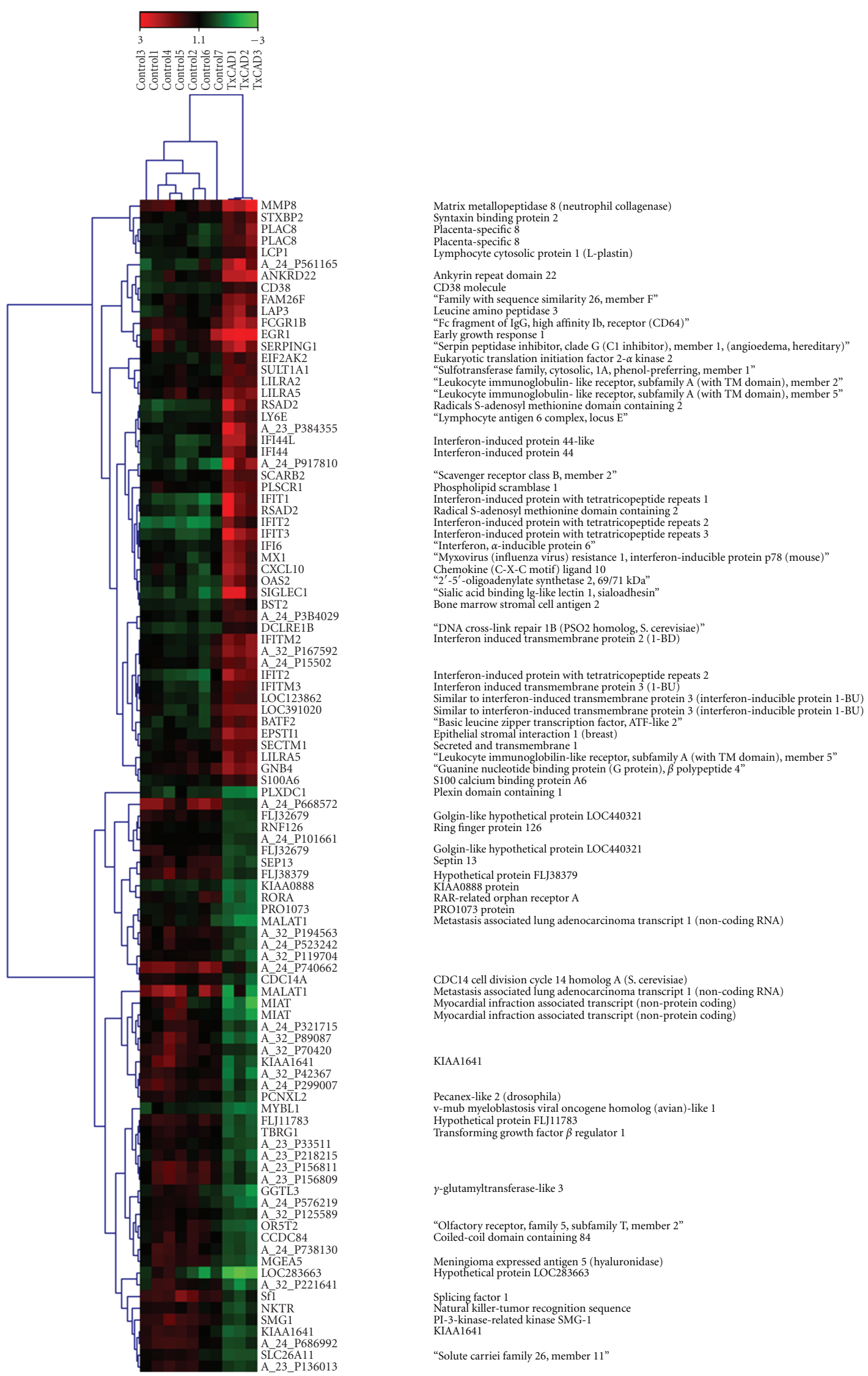

"Solute carriei family 26, member 11"

FIGURE 1: Clustered heat map of top 100 up- and down-regulated genes in patients with advanced transplant coronary artery disease and controls. Gene symbol and gene name are provided when available. Platform unique identifier is provided otherwise. 


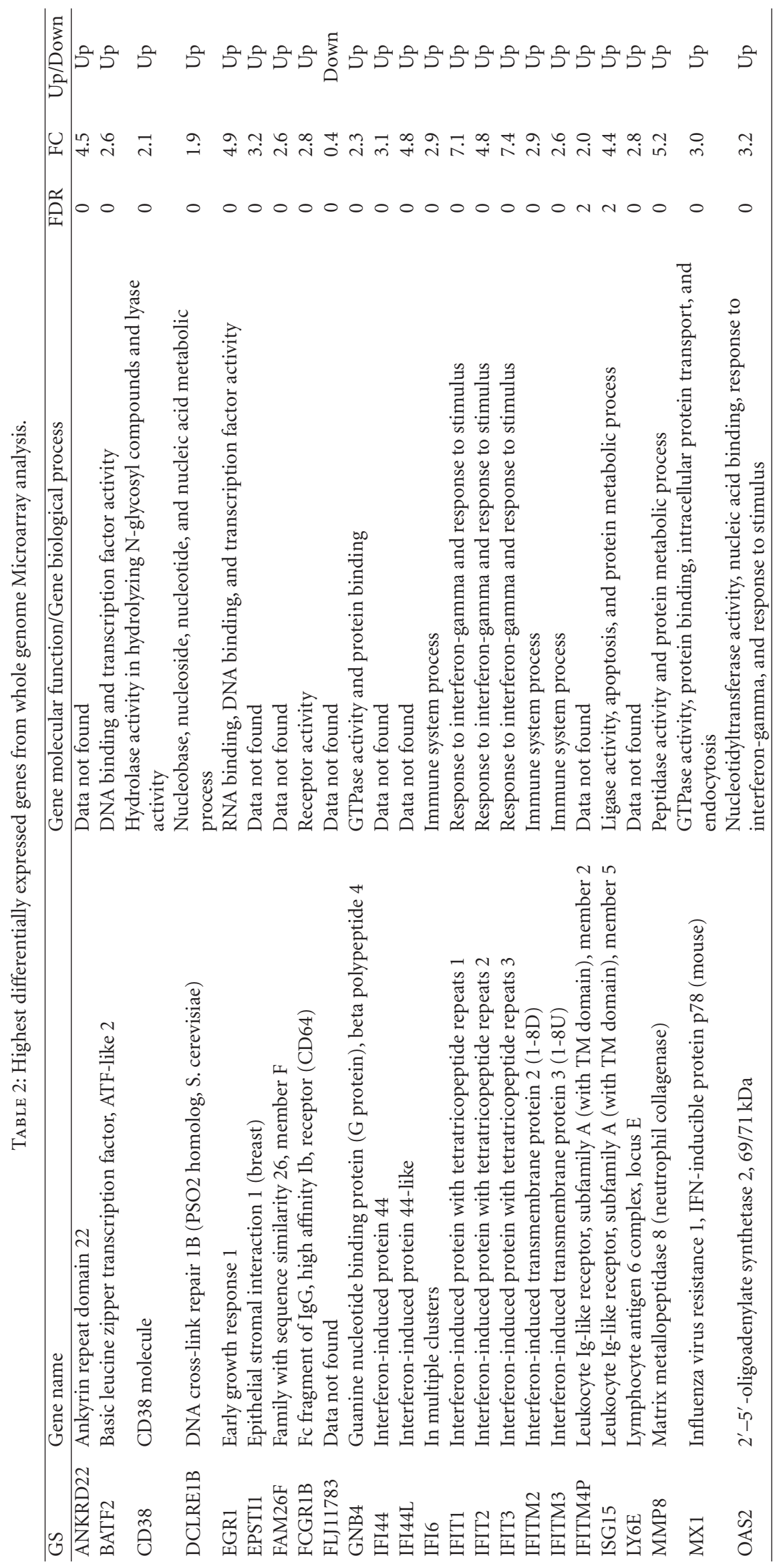




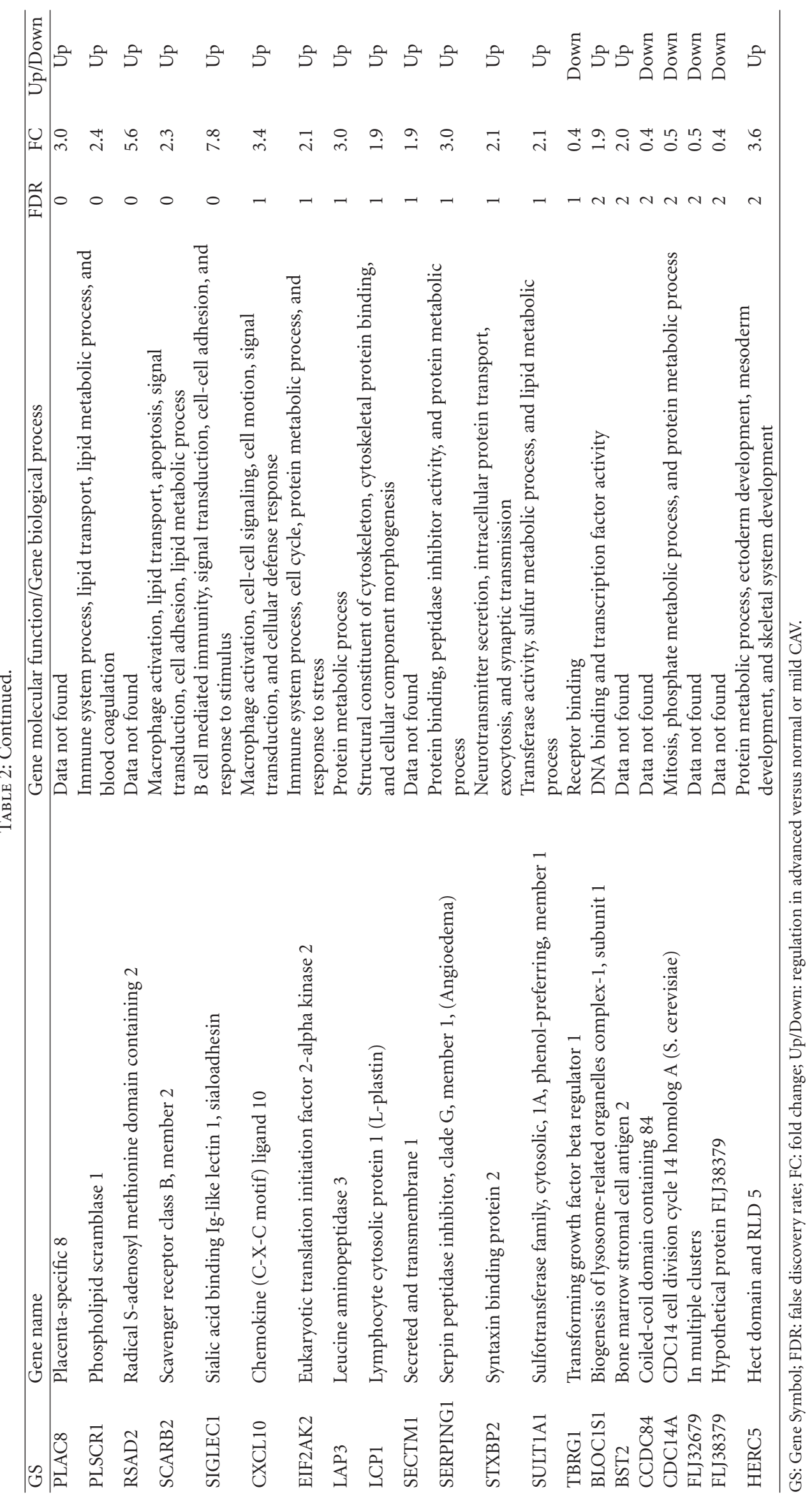


TABLE 3: Gene ontology categories enriched by genes expressed differentially between patients with and without advanced CAV.

\begin{tabular}{|c|c|c|c|}
\hline Regulation in Adv-CAV & $\begin{array}{l}\text { Number of } \\
\text { genes }\end{array}$ & GO category type & Exemplary GO categories \\
\hline \multirow[t]{23}{*}{ Up-/Down-regulated } & 291 & Biological process & GO:0006955 immune response \\
\hline & & & GO:0002376 immune system process \\
\hline & & & GO:0051707 response to other organism \\
\hline & & & GO:0009607 response to biotic stimulus \\
\hline & & & GO:0006954 inflammatory response \\
\hline & & & GO:0050896 response to stimulus \\
\hline & & & GO:0009615 response to virus \\
\hline & & & GO:0009611 response to wounding \\
\hline & & & GO:0006952 defense response \\
\hline & & & GO:0045087 innate immune response \\
\hline & & & GO:0009605 response to external stimulus \\
\hline & & & GO:0042116 macrophage activation \\
\hline & & & GO:0006950 response to stress \\
\hline & & Molecular function & $\begin{array}{l}\text { GO: } 0045408 \text { regulation of interleukin- } 6 \text { biosynthetic } \\
\text { process }\end{array}$ \\
\hline & & & GO:0007249 I-kappaB kinase NF-kappaB cascade \\
\hline & & & GO:0007243 protein kinase cascade \\
\hline & & & $\begin{array}{l}\text { GO:0007009 plasma membrane organization and } \\
\text { biogenesis }\end{array}$ \\
\hline & & & GO:0032635 interleukin-6 production \\
\hline & & & GO:0042226 interleukin- 6 biosynthetic process \\
\hline & & & $\begin{array}{l}\text { GO: } 0045410 \text { positive regulation of interleukin- } 6 \\
\text { biosynthetic process }\end{array}$ \\
\hline & & & GO:0009057 macromolecule catabolic process \\
\hline & & & GO:0009595 detection of biotic stimulus \\
\hline & & & GO:0007250 activation of NF-kappaB-inducing kinase \\
\hline \multirow[t]{2}{*}{ Up-Regulated } & 170 & Biological process & None \\
\hline & & Molecular function & None \\
\hline \multirow[t]{18}{*}{ Down-Regulated } & 123 & Biological process & GO:0006955 immune response \\
\hline & & & GO:0002376 immune system process \\
\hline & & & GO:0051707 response to other organism \\
\hline & & & GO:0009607 response to biotic stimulus \\
\hline & & & GO:0006954 inflammatory response \\
\hline & & & GO:0050896 response to stimulus \\
\hline & & & GO:0009615 response to virus \\
\hline & & & GO:0009611 response to wounding \\
\hline & & & GO:0006952 defense response \\
\hline & & & GO:0045087 innate immune response \\
\hline & & & GO:0009605 response to external stimulus \\
\hline & & & GO:0042116 macrophage activation \\
\hline & & & GO:0006950 response to stress \\
\hline & & & GO:0048518 positive regulation of biological process \\
\hline & & & GO:0002250 adaptive immune response \\
\hline & & Molecular function & $\begin{array}{l}\text { GO: } 0045408 \text { regulation of interleukin- } 6 \text { biosynthetic } \\
\text { process }\end{array}$ \\
\hline & & & GO:0007249 I-kappaB kinase NF-kappaB cascade \\
\hline & & & GO:0007243 protein kinase cascade \\
\hline
\end{tabular}


Table 3: Continued.

\begin{tabular}{lll}
\hline $\begin{array}{l}\text { Regulation in Adv-CAV } \\
\text { genes }\end{array}$ & $\begin{array}{c}\text { Go category type } \\
\text { Exemplary GO categories }\end{array}$ \\
\hline & $\begin{array}{l}\text { GO:0007009 plasma membrane organization and } \\
\text { biogenesis }\end{array}$ \\
GO:0032635 interleukin-6 production \\
GO:0042226 interleukin-6 biosynthetic process \\
GO:0045410 positive regulation of interleukin-6 \\
biosynthetic process \\
GO:0009057 macromolecule catabolic process \\
GO:0009595 detection of biotic stimulus \\
GO:0007250 activation of NF-kappaB-inducing kinase \\
GO:0045084 positive regulation of interleukin-12 \\
biosynthetic process
\end{tabular}

Adv-CAV: advanced cardiac allograft vasculopathy.

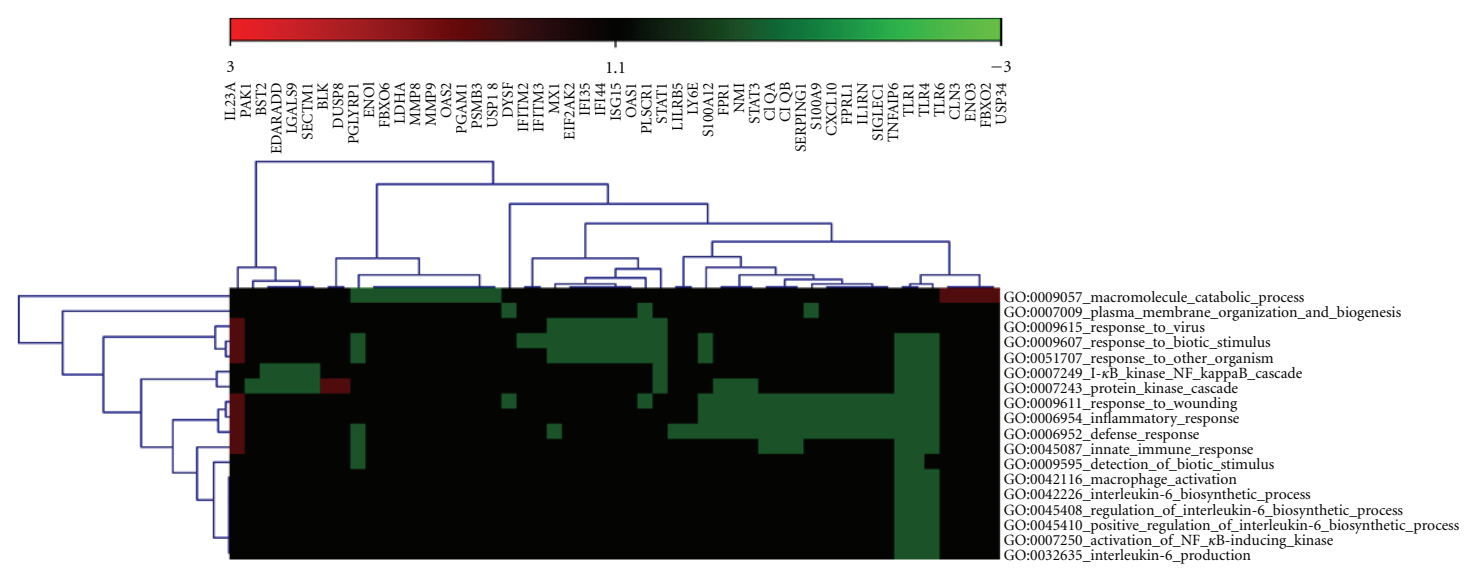

FIGURE 2: Clustered heat map of enriched GO categories by differentially expressed genes. Instances of cross-talk are shown that links GO categories enriched by similar genes. The green areas show GO categories enriched by the down-regulated genes while small red areas in the corners show GO categories enriched by up-regulated genes.

TABLE 4: PCR corroboration of differentially expressed genes and Microarray-based FDR.

\begin{tabular}{lcccc}
\hline Gene & Up/Down & FC & $P$-value (PCR) & FDR (Microarray) \\
\hline FPRL1 & up & 2.3 & .005 & 3 \\
S100A9 & up & 2.9 & .005 & 3.06 \\
CXCL10b & up & 3.4 & .009 & 1.41 \\
CXCL10a & up & 3.4 & .019 & 1.41 \\
PRO1073 & down & 0.5 & .045 & 2.35 \\
MMP9 & up & 8.0 & .045 & 3.13 \\
ENO1 & up & 1.7 & .083 & 4.60 \\
FAS & up & 1.8 & .104 & 4.17 \\
\hline
\end{tabular}

of a disease under investigation. These disease specific gene signatures can be used to select patients at a specific state of a disease with an accuracy that facilitates the diagnosis of the disease and selection of patients for different treatment options.
In this pilot study, patients with advanced CAV show peripheral blood gene expression profiles that differ already in the early period after transplantation from those of patients who will not develop advanced CAV. The findings are consistent with previous suggestions that donor- and recipient-related factors in the perioperative period may play major roles in the immune response and development of a sustained chronic response to immune injury, leading to proliferation of the endovascular matrix, with consequent obstruction of the blood flow and impairment of allograft function $[2,4,9,10]$.

In previous studies, gene expression profiles of PBMC have been shown to correlate with presence or absence of concurrent acute cellular rejection [16-18, 24], antibody mediated rejection [19], and in response to mechanical circulatory support device implantation [25, 26]. Based on these gene expression profiles, the genomic classifier developed to rule out acute cellular cardiac allograft rejection has also been shown to correlate with different organ function related parameters of rejection $[27,28]$, and 
longitudinal changes in clinical profiles $[29,30]$. In current study, we hypothesized that gene signatures early after HTx correlate with future development of CAV. To the best of our knowledge, this is the first report of using a high throughput genomic screening approach to identify patients at risk of developing advanced CAV on the basis of leukocyte samples obtained during the early period after transplantation. This observation has potentially important implications.

Recently CAV has been reported as one of the major causes of new-onset graft dysfunction and associated outcomes [31]. Early detection of CAV was proposed as the key strategy, to identify surrogate markers for late cardiac allograft outcomes [1]. Currently, the most accurate diagnostic test to define the phenotype of CAV is intravascular ultrasound [9]. Evaluation of the neointimal proliferation rate between months 3 and 12 has been shown to predict survival and major adverse cardiac events at long-term follow-up [11] but intravascular ultrasound is invasive, expensive and complication prone. Therefore, early profiling of an immune response that differentiates a "high CAVrisk phenotype" from a "low CAV-risk phenotype" may become valuable to early identify these patients, model timely therapeutic interventions and monitor response.

This concept in HTx is not new. Earlier observations in animal models of rejection suggested that "unstable tolerance induction" is associated with the persistent immune activation that mediates destruction of graft parenchymal cells, and that the evaluation of biological processes involved might be useful in identifying different types of rejection and the likelihood of progression to chronic forms [32].

In our study, we found differentially expressed genes that significantly enriched GO categories including the innate immunity, macrophage activation, interleukin-6, activation of the NF-KappaB cascade and response to virus. The finding that the innate immune response plays an important role is interesting because it has been already described in the literature. Differentially expressed genes, among others in this category, included Toll-like receptors 1, 4 and 6, and Interleukin-23 alpha. Several authors proposed an important role of innate immunity in the pathogenesis of CAV [10,3335]. For example, it has been shown that in patients with allograft endothelial dysfunction (an early clinical indicator of transplant vasculopathy), mRNA transcript level and surface expression of TLR-4 on circulating monocyte is significantly higher than controls [34]. According to our results the categories related to these mechanisms were enriched by the down-regulated genes. The interaction between the up- and down-regulated genes collectively determines the functioning of different biological processes as assessed by high through-put microarray analysis. Our observations show some interesting expression patterns of these mechanisms during the early period post-HTx which needs further understanding. Therapy with Simvastatin that inhibits allograft inflammatory activity and attenuates endothelial coronary dysfunction shows reduced trans-cardiac IL-6 and TNF-alpha gradients [33] and polymorphisms within the promoter region of the IL- 6 gene has been proposed as risk markers for CAV [36]. In addition, the transcription factor NFKB becomes up-regulated in response to CD40/ CD40 ligand mediating apoptosis of endothelial cells. Inhibition of this pathway has been shown to be protective against the development of CAV [37]. The GO analysis shows that the STAT1 gene involved in the NFKB pathway is also part of the response to virus showing a "cross-talk" between both categories and suggesting that mechanisms of immune damage related to viruses (i.e., CMV) $[9,10]$ may be related to the NFKB pathway.

The findings presented in this paper should encourage the development of strategies to advance evaluation and management of heart transplant recipients in a preventative, preemptive and personalized way, but the conclusion from this pilot study should be interpreted within the important limitations imposed by the small sample size, the use of angiography which is an imperfect standard for detection of CAV, and the known variable reproducibility of gene expression studies [38]. Corroboration of genes was based on retrospective PCR data. Only a small number of genes were available that were present in the Microarray-based candidate gene list.

In conclusion, our data show that peripheral blood leukocyte genes, specifically innate immune response genes, are differentially expressed during the early time after HTx in patients who develop advanced CAV. Larger prospectively designed studies are needed to corroborate our findings.

\section{Disclosure}

All authors report no conflict of interests in relationship to the data presented here.

\section{Acknowledgments}

This research was supported in part by the Intramural Research Program of the NIH, National Cancer Institute, Center for Cancer Research (BZ), and research support from XDx Inc. Brisbane, CA (TK). K. Shahzad and M. Cadeiras contributed equally for primary authorship.

\section{References}

[1] M. R. Mehra, R. Benza, M. C. Deng, S. Russell, and S. Webber, "Surrogate markers for late cardiac allograft survival," American Journal of Transplantation, vol. 4, no. 7, pp. 11841191, 2004.

[2] J. Compston and E. Shane, Bone Disease of Organ Transplantation, Elsevier Academic Press, Burlington, Mass, USA, 2005.

[3] C. A. Labarrere, D. Pitts, D. R. Nelson, and W. P. Faulk, "Vascular tissue plasminogen activator and the development of coronary artery disease in heart-transplant recipients," The New England Journal of Medicine, vol. 333, no. 17, pp. 11111116, 1995.

[4] C. A. Labarrere, D. R. Nelson, C. J. Cox, D. Pitts, P. Kirlin, and H. Halbrook, "Cardiac-specific troponin I levels and risks of coronary artery disease and graft failure following heart transplantation," Journal of the American Medical Association, vol. 284, no. 4, pp. 457-464, 2000.

[5] M. R. Mehra, H. O. Ventura, A. Escobar, C. A. Cassidy, F. W. Smart, and D. D. Stapleton, "Does donor and recipient sex influence the development of cardiac allograft vasculopathy?" 
Transplantation Proceedings, vol. 27, no. 3, pp. 1926-1929, 1995.

[6] M. H. Yamani, E. M. Tuzcu, R. C. Starling et al., "Myocardial ischemic injury after heart transplantation is associated with upregulation of vitronectin receptor $(\alpha \mathrm{v} \beta 3)$, activation of the matrix metalloproteinase induction system, and subsequent development of coronary vasculopathy," Circulation, vol. 105, no. 16, pp. 1955-1961, 2002.

[7] K. Pethig, B. Heublein, I. Kutschka, and A. Haverich, "Systemic inflammatory response in cardiac allograft vasculopathy: high-sensitive C-reactive protein is associated with progressive luminal obstruction," Circulation, vol. 102, no. 19, supplement 3, pp. III233-III236, 2000.

[8] C. A. Labarrere, D. R. Nelson, and K. L. Spear, "Nonimmunologic vascular failure of the transplanted heart," Journal of Heart and Lung Transplantation, vol. 22, no. 3, pp. 236-240, 2003.

[9] S. P. Pinney and D. Mancini, "Cardiac allograft vasculopathy: advances in understanding its pathophysiology, prevention, and treatment," Current Opinion in Cardiology, vol. 19, no. 2, pp. 170-176, 2004.

[10] D. Schmauss and M. Weis, "Cardiac allograft vasculopathy: recent developments," Circulation, vol. 117, no. 16, pp. 21312141, 2008.

[11] J. A. Kobashigawa, J. M. Tobis, R. C. Starling et al., "Multicenter intravascular ultrasound validation study among heart transplant recipients: outcomes after five years," Journal of the American College of Cardiology, vol. 45, no. 9, pp. 1532-1537, 2005.

[12] D. O. Taylor, L. B. Edwards, M. M. Boucek et al., "Registry of the International Society for Heart and Lung Transplantation: twenty-fourth official adult heart transplant report-2007," Journal of Heart and Lung Transplantation, vol. 26, no. 8, pp. 769-781, 2007.

[13] P. R. Rickenbacher, F. J. Pinto, N. P. Lewis et al., "Prognostic importance of intimal thickness as measured by intracoronary ultrasound after cardiac transplantation," Circulation, vol. 92, no. 12 , pp. 3445-3452, 1995.

[14] W. T. Evanochko, S. D. Buchthal, J. A. den Hollander et al., "Cardiac transplant patients response to the 31P MRS stress test," Journal of Heart and Lung Transplantation, vol. 21, no. 5, pp. 522-529, 2002.

[15] G. Sigurdsson, P. Carrascosa, M. H. Yamani et al., "Detection of transplant coronary artery disease using multidetector computed tomography with adaptative multisegment reconstruction," Journal of the American College of Cardiology, vol. 48, no. 4, pp. 772-778, 2006.

[16] P. A. Horwitz, E. J. Tsai, M. E. Putt et al., "Detection of cardiac allograft rejection and response to immunosuppressive therapy with peripheral blood gene expression," Circulation, vol. 110, no. 25, pp. 3815-3821, 2004.

[17] M. Schoels, T. J. Dengler, R. Richter, S. C. Meuer, and T. Giese, "Detection of cardiac allograft rejection by realtime PCR analysis of circulating mononuclear cells," Clinical Transplantation, vol. 18, no. 5, pp. 513-517, 2004.

[18] M. C. Deng, H. J. Eisen, M. R. Mehra et al., "Noninvasive discrimination of rejection in cardiac allograft recipients using gene expression profiling," American Journal of Transplantation, vol. 6, no. 1, pp. 150-160, 2006.

[19] M. Cadeiras, M. Bayern, E. Burke et al., "Gene expression profiles of patients with antibody-mediated rejection after cardiac transplantation," Journal of Heart and Lung Transplantation, vol. 27, no. 8, pp. 932-934, 2008.
[20] V. G. Tusher, R. Tibshirani, and G. Chu, "Significance analysis of microarrays applied to the ionizing radiation response," Proceedings of the National Academy of Sciences of the United States of America, vol. 98, no. 9, pp. 5116-5121, 2001.

[21] J. N. Weinstein, T. G. Myers, P. M. O’Connor et al., “An information-intensive approach to the molecular pharmacology of cancer," Science, vol. 275, no. 5298, pp. 343-349, 1997.

[22] B. R. Zeeberg, H. Qin, S. Narasimhan et al., "High-throughput GoMiner, an 'industrial-strength' integrative gene ontology tool for interpretation of multiple-microarray experiments, with application to studies of Common Variable Immune Deficiency (CVID)," BMC Bioinformatics, vol. 6, article 168, 2005.

[23] M. A. Harris, J. Clark, A. Ireland et al., "The Gene Oncology (GO) database and informatics resource," Nucleic Acids Research, vol. 32, database issue, pp. D258-D261, 2004.

[24] M. Cadeiras, M. von Bayern, A. Sinha et al., "Drawing networks of rejection-a systems biological approach to the identification of candidate genes in heart transplantation," Journal of Cellular and Molecular Medicine. In press.

[25] A. Sinha, K. Shahzad, F. Latif et al., "Peripheral blood mononuclear cell transcriptome profiles suggest T-cell immunosuppression after uncomplicated mechanical circulatory support device surgery," Human Immunology, vol. 71, no. 2, pp. 164-169, 2010.

[26] K. Shahzad, R. N. Sladen, F. Latif et al., "Leukocyte gene expression signatures of multiorgan dysfunction after mechanical circulatory support device implantation in patients with heart failure," Circulation, vol. 122, no. 21, 2010, abstract no. A13840.

[27] M. Cadeiras, K. Shahzad, M. M. John et al., "Relationship between a validated molecular cardiac transplant rejection classifier and routine organ function parameters," Clinical Transplantation, vol. 24, no. 3, pp. 321-327, 2010.

[28] K. Shahzad, J. He, Q. Li, Q. A. Aziz, and M. C. Deng, "Relationship between prolonged QTc interval, cardiac allograft dysfunction and elevated molecular gene expression profiling test score after heart transplantation," Journal of Heart and Lung Transplantation, vol. 29, no. 6, pp. 711-713, 2010.

[29] M. C. Deng, G. Alexander, H. Wolters et al., "Low variability of intraindividual longitudinal leukocyte gene expression profiling cardiac allograft rejection scores," Transplantation, vol. 90, no. 4, pp. 459-461, 2010.

[30] K. Shahzad, M. Cadeiras, K. Arai, D. Abramov, E. Burke, and M. C. Deng, "Unexplained graft dysfunction after heart transplantation-role of novel molecular expression test score and QTc-interval: a case report," Cardiology Research and Practice, vol. 2010, Article ID 230810, 2010.

[31] K. Shahzad, Q. A. Aziz, J.-P. Leva et al., "New-onset graft dysfunction after heart transplantation-incidence and mechanism-related outcomes," Journal of Heart and Lung Transplantation. In press.

[32] A. Shimizu, K. Yamada, D. H. Sachs, and R. B. Colvin, "Intragraft events preceding chronic renal allograft rejection in a modified tolerance protocol," Kidney International, vol. 58, no. 6, pp. 2546-2558, 2000.

[33] M. Weis, S. Pehlivanli, B. M. Meiser, and W. Von Scheidt, "Simvastatin treatment is associated with improvement in coronary endothelial function and decreased cytokine activation in patients after heart transplantation," Journal of the American College of Cardiology, vol. 38, no. 3, pp. 814-818, 2001. 
[34] H. Methe, E. Zimmer, C. Grimm, M. Nabauer, and J. Koglin, "Evidence for a role of toll-like receptor 4 in development of chronic allograft rejection after cardiac transplantation," Transplantation, vol. 78, no. 9, pp. 1324-1331, 2004.

[35] J. Obhrai and D. R. Goldstein, "The role of toll-like receptors in solid organ transplantation," Transplantation, vol. 81, no. 4, pp. 497-502, 2006.

[36] C. G. Densem, M. Ray, I. V. Hutchinson, N. Yonan, and N. H. Brooks, "Interleukin-6 polymorphism: a genetic risk factor for cardiac transplant related coronary vasculopathy?" Journal of Heart and Lung Transplantation, vol. 24, no. 5, pp. 559-565, 2005.

[37] C. R. Longo, M. B. Arvelo, V. I. Patel et al., "A20 protects from CD40-CD40 ligand-mediated endothelial cell activation and apoptosis," Circulation, vol. 108, no. 9, pp. 1113-1118, 2003.

[38] M. C. Deng, H. J. Eisen, and M. R. Mehra, "Methodological challenges of genomic research-the Cargo study," American Journal of Transplantation, vol. 6, no. 5, part 1, pp. 1086-1087, 2006. 


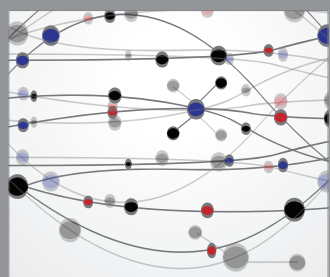

The Scientific World Journal
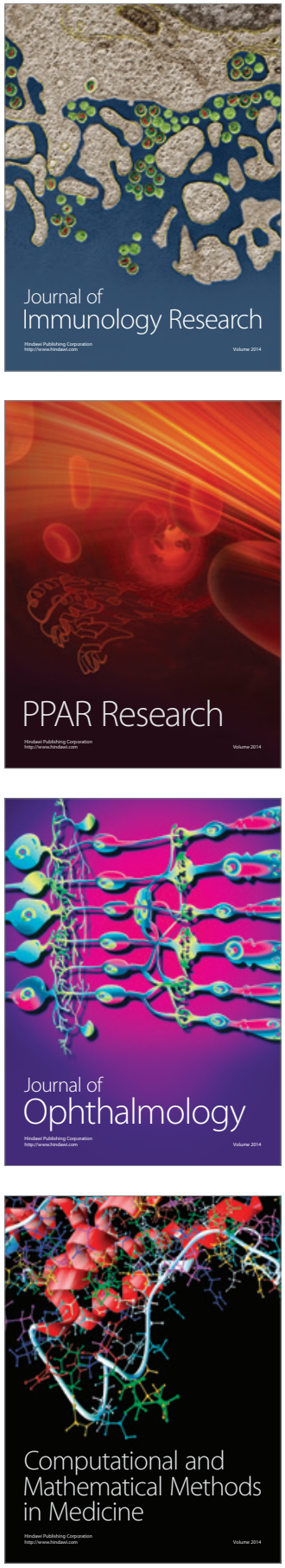

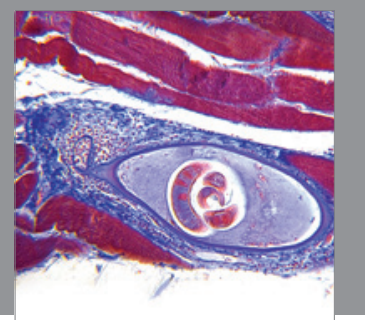

Gastroenterology

Research and Practice
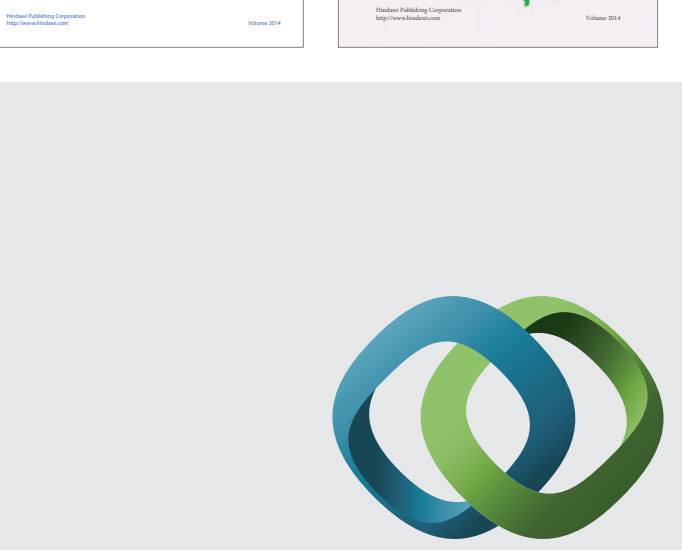

\section{Hindawi}

Submit your manuscripts at

http://www.hindawi.com
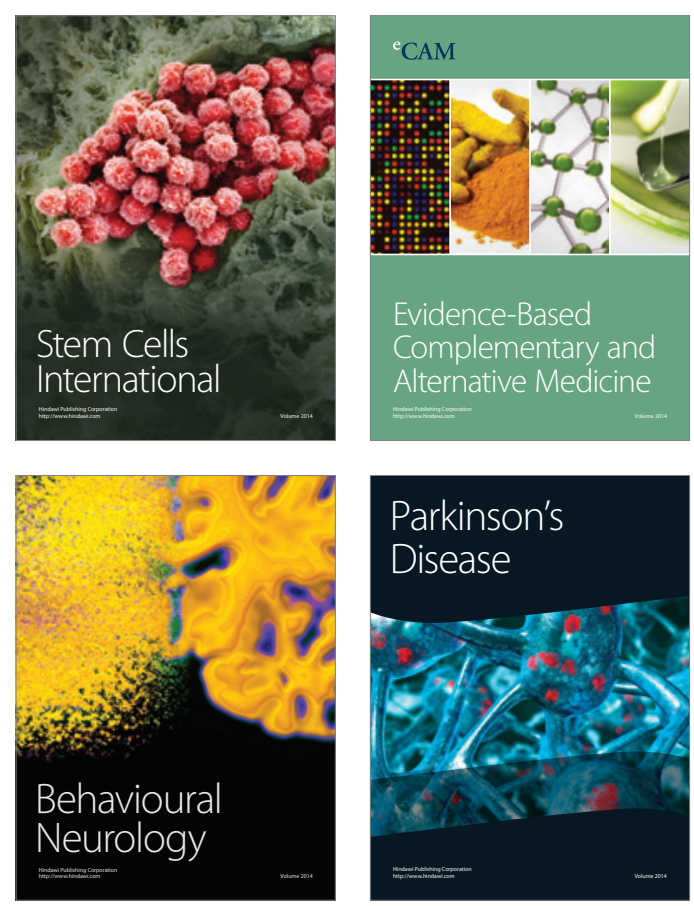

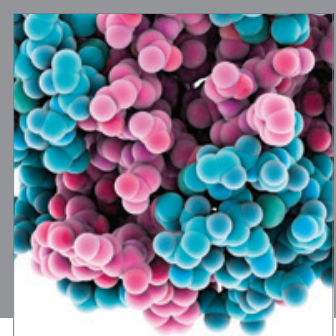

Journal of
Diabetes Research

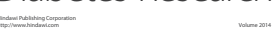

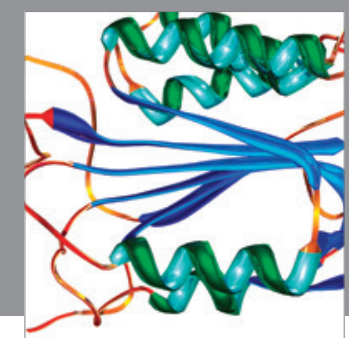

Disease Markers
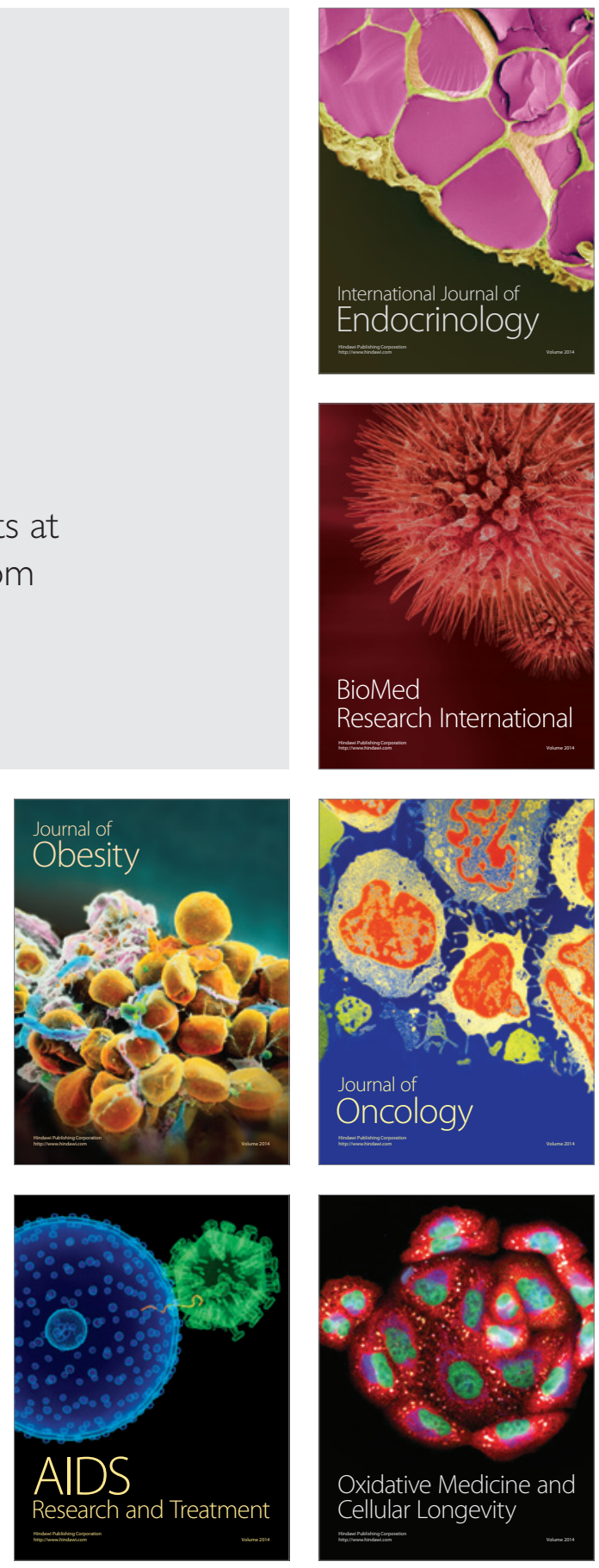